Radiologe 2010 · 50:5

DOI 10.1007/s00117-009-1964-2

Online publiziert: 24. Dezember 2009

(c) Springer-Verlag 2009

\author{
M. Simon \\ Klinik für Radiologie und Nuklearmedizin, \\ Universitätsklinikum Schleswig-Holstein, Campus Lübeck, Lübeck
}

\title{
Unverbesserlich strukturiert - oder kann der Computer den Befund verbessern?
}

hernd konstant blieben (Richtigkeit 91,4 bzw. 92,4 und Vollständigkeit 67,8 bzw. $71,7)$, rutschten die strukturierten Kollegen tief nach unten. Die Richtigkeit sank von 91,5 auf 88,7, die Befundvollständigkeit erschreckend von 68,7 auf 54,3.

Wie? Zumindest vollständiger hätten die strukturierten Befunde bitteschön sein müssen.

Nein, verlesen haben wir uns nicht: Zumindest diese Software hat hier nichts gebracht, und das Hirn ist ein besserer Computer als manche vermuten. Verallgemeinerungen hingegen sind unzulässig, zumal der Artikel auch nicht ganz klar zu erkennen gibt, ob es sich bei der Anwendung um eine Befundungsoberfläche handelt, die verschiedene Kriterien und Textvorlagen zur Auswahl gibt, oder um ein vollständiges SR-System nach DICOM-Kriterien.

Die Computeranwendung kann letztlich nicht besser sein als ihr Schöpfer, d. h. es muss vorab anatomisch und pathologisch vollständig sein. Vermuten muss man, dass dies hier nicht der Fall war und es mehr an dem getesteten System selbst als an der SR-Befundung im Allgemeinen liegt. Nun, immerhin ein Anlass zu prüfen, ob es auch besser geht...!

Ein Nebenergebnis zum Schmunzeln gab es auch noch: Da nun schon mal verschiedene Weiterbildungsjahre angetreten waren, konnte man auch innerhalb dieser den direkten Vergleich antreten. Bezogen auf die Befundrichtigkeit lag das 3. Jahr vorn, bezogen auf die Vollständigkeit das 2. Jahr; die Kollegen des 4. Jahres bildeten unabhängig von der Art der Befundung das Schlusslicht. Auch Über- schätzung der eigenen Routine ist der Befundqualität abträglich, auch wenn man schon vorher wusste, wonach man Ausschau halten sollte... Da gab es die Phasen des Lernens: Die berechtigte Unsicherheit, die unberechtigte Sicherheit und schließlich die gerechtfertigte Sicherheit. Und zuletzt, bei ehrlicher Einsicht, die unberechtigte Unsicherheit, die den wirklich Erfahrenen auszeichnet.

\section{Korrespondenzadresse \\ Dr. M. Simon}

Klinik für Radiologie und Nuklearmedizin, Universitätsklinikum Schleswig-Holstein, Campus Lübeck,

Ratzeburger Allee 160, 23538 Lübeck

Martin.Simon@uk-sh.de

Interessenkonflikt. Der korrespondierende Autor weist auf folgende Beziehung hin: Research Grant IMAGE Information Systems Europe Ltd. 